CARDIOLOGY UPDATE

\title{
Cardiac magnetic resonance imaging
}

\author{
G J Heatlie, K Pointon
}

Postgrad Med J 2004;80:19-22. doi: 10.1136/pgmi.2003.010355

Recent advances in technology have allowed magnetic resonance imaging to be exploited in cardiology. It is capable of giving high definition structural and functional information. The current applications of this technique and how it may develop in the future are reviewed.

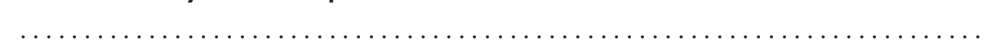

M

agnetic resonance imaging (MRI) has been in clinical use for 20 years and has a major role in the fields of musculoskeletal and neurological imaging. Progress in technology has reduced scan times and improved image resolution, and the modality now can offer static and dynamic cardiac and vascular imaging on a wide scale.

Cardiac MRI should be regarded as complementary to other imaging modalities. The availability of transthoracic and transoesophageal echocardiography, their portability, and the experience in this field will keep them as first line investigations for many years. Cardiac MRI cannot measure coronary artery calcification and has not achieved the spatial resolution of the most modern computed tomography systems. Widespread use of computed tomography and nuclear medicine for multiple follow up cardiac studies is limited by the cumulative radiation doses acquired. MRI is currently of particular cardiac anomalies and in visualising abnormalities of the aorta. There is a rapidly expanding role of MRI in the assessment of ischaemic heart disease with myocardial perfusion and scar imaging. morphological cardiac imaging is similar to that used for other applications and many of the modern systems will have pre-loaded software able to perform some static and cine imaging. This technology is available from dedicated research centres through to the district general hospital.

Absolute contraindications to cardiac MRI are few but include permanent pacemakers, defibrillators, and cerebral aneurysm clips. Most prosthetic heart valves and coronary artery stents are safe, although they may produce some artefact.

See end of article for authors' affiliations

Correspondence to: Dr Grant Heatlie, Queens Medical Centre, Derby Road, Nottingham NG7 2UH, UK; grant.heatlie@ virgin.net

Submitted 25 May 2003 Accepted 23 June 2003

.................... quantum mechanics called "spin", which can be thought of as angular momentum, momentum associated with the spinning motion of a rotating body. Some nuclei with an even number of nucleons have zero overall spin, while all nuclei with an odd number of nucleons will have value in assessing following up congenital

The strength of magnet required to achieve

net spin. Nuclei carry a charge and so, according to Faraday's law, each of these moving nuclei has a net magnetic moment as they are moving charges. Hydrogen atoms are a major constituent of the body and are the nuclei normally imaged by cardiac MRI since their magnetic moment is high. Other atoms can be used but provide poor anatomical detail because of their limited distribution within tissues. Since nuclei normally have random motion, the components of magnetic moment cancel out and there is no overall magnetic field associated with matter.

When nuclei with non-zero spin are put in an external magnetic field (B), they try to align themselves parallel to the external field as this is the lowest energy state available to them. They are unable to do this exactly and instead rotate around the axis of the external magnetic field. This is analogous to a child's spinning top rotating around a vertical axis as it spins. Since the magnetic moments of the nuclei are no longer randomly aligned, they add together to form a net magnetic field from the tissue parallel to the external field, but much smaller in magnitude. The size of this field is small compared with the background magnetic field, but the technique of cardiac MRI is capable of detecting this magnetisation.

The principle of the MRI scanner is simple. A large superconducting magnet produces a strong external magnetic field, typically 1.5 teslas, about 1500 times the strength of the earth's magnetic field. This magnetic field is not quite uniform but has small variations in field strength related to position within the magnetic field; this effectively allows the position of nuclei to be mapped. Electromagnetic energy is transmitted from a coil to the nuclei at the resonant frequency (which is the nuclei's "natural" frequency allowing maximal energy absorbtion) exciting them to a higher energy state. The net magnetic moment in this higher energy state moves out of the direction of $\mathrm{B}$. As this decays back to a lower energy state (parallel to B) then energy is released as an electromagnetic wave. This is detected by an aerial or body coil. The received frequency depends on the local field strength so that the intensity and spatial location can be mapped. Different tissues return to their lower energy states at different rates and so tissues can be differentiated. The received signal can be reconstructed using a mathematical technique called a Fourier transformation and powerful computing to give an MRI image.

Abbreviations: ECG, electrocardiogram; MRI, magnetic resonance imaging 


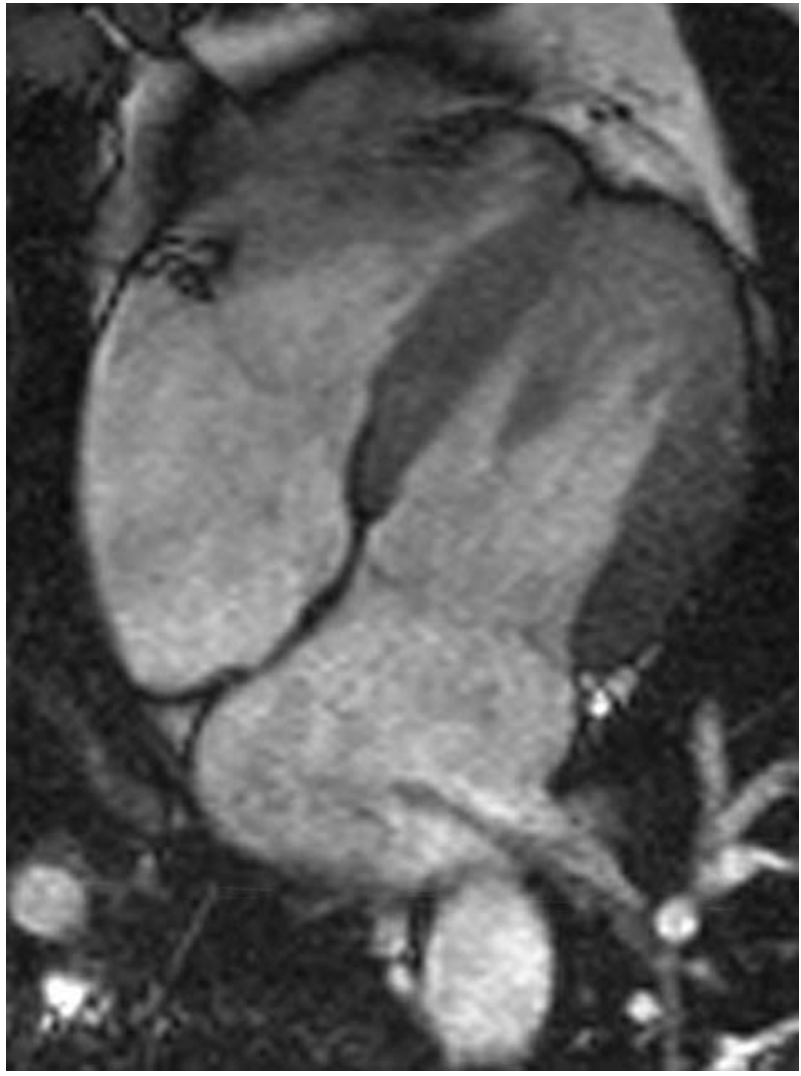

Figure 1 A four chamber view of the heart during systole. The right ventricle lies anterior (towards the top of the picture).

There are three fundamental forms of MRI image used: black blood imaging (spin echo) which produces detailed anatomical images; white blood imaging (gradient echo) which produces information on flow and function; and velocity mapping, a technique not dissimilar to Doppler. Although each is produced using the generic principle described above, a different image emerges from each of these techniques due to the application of different sequences-that is, different combinations of radiofrequency pulses and timings of the returned signal. Magnets tend to be dedicated either to anatomical or functional applications, as the field strength and magnetic gradients used differ.

\section{APPLICATIONS OF CARDIAC MRI}

\section{Myocardial mass and volume}

The most frequent requirement of any imaging modality is to assess left ventricular function and dimensions. Quantifying left ventricular function provides a powerful prognostic index for patients with heart failure and guides the treatment that they are offered.'

Echocardiography, the standard technique for measuring chamber volumes, may be hindered by technical problems such as poor echo windows, poor endocardial definition, and inaccuracies in calculation related to the geometric assumptions needed to derive three dimensional estimates from two dimensional measurements. Nuclear ventriculography is free from many of these problems but involves radiation exposure.

Standard MRI is a robust method of measuring volume: three dimensional ventricular images can be obtained at specific points in the cardiac cycle using the electrocardiogram $(E C G)^{2}$ to derive reproducible and accurate left and right ventricular function, myocardial volume, and mass.

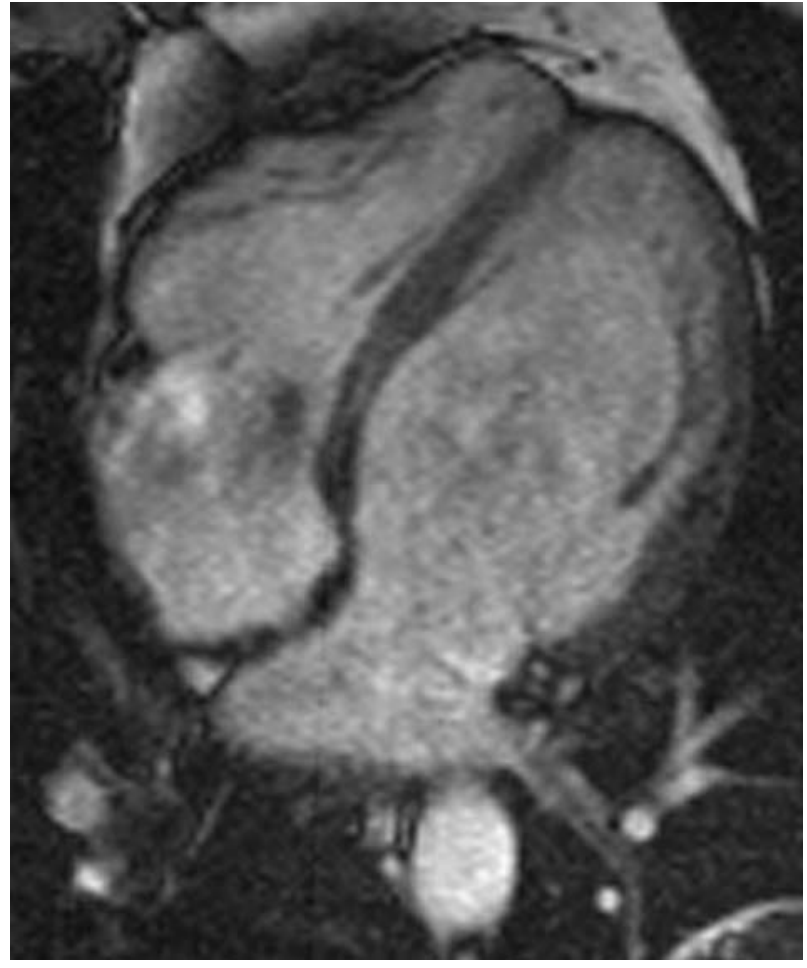

Figure 2 A four chamber view of the heart during diastole. A series of these images at different levels can be used to calculate the systolic and diastolic volume of the heart and so estimate ejection fraction.

Distorted areas of wall thinning and aneurysms relating to previous myocardial infarction can be demonstrated. Cine mode MRI allows regional and global systolic function to be evaluated and wall motion abnormalities to be identified as epicardial and endocardial definition are excellent.

\section{Flow}

The amplitude and phase of the returning magnetic resonance radio signal contains information about blood velocity. Two dimensional flow maps can be derived and

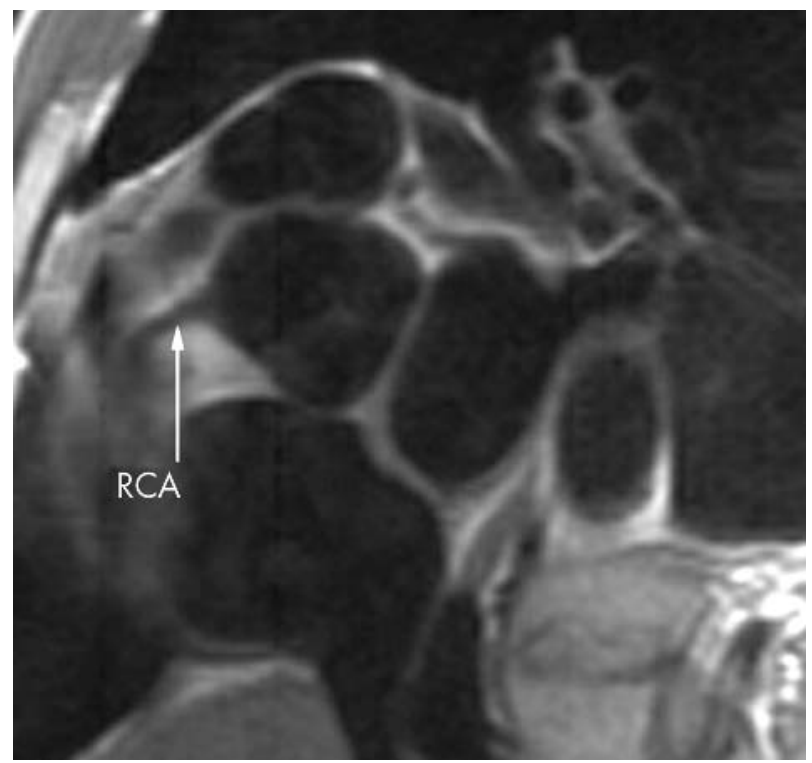

Figure 3 The proximal lumen of the right coronary artery is clearly seen. 
correlated with the ECG to give rates of change of velocity through the cardiac cycle. Combining velocity with vessel or valve area means flow can be derived. Thus real time measurements of anatomy and flow volumes are acquired together.

Echo Doppler will in most cases be adequate to assess valvular disease. MRI quantification of stenotic and regurgitant lesions can be helpful in specific situations. For example, it is difficult to estimate the severity of an eccentric mitral regurgitant jet of blood using standard echo criteria. MRI in this situation is able to more reliably quantify regurgitation, because the imaging planes are not as limited. ${ }^{3}$

\section{Coronary artery imaging}

An exciting potential application of magnetic resonance imaging is as a screening tool for the presence of coronary artery disease, allowing invasive angiography to be reserved as a prelude to coronary intervention.

In order to obtain satisfactory images of the coronary arteries non-invasively, very accurate image registration and fine resolution are essential. Cardiac motion in systole and diastole cause the heart both to shorten and rotate, further complicated by respiration. Imaging of the origins and proximal portions of the main coronary arteries and bifurcation is possible, but more distal portions are less reliably seen. ${ }^{4}$

Coronary artery imaging is currently restricted to the proximal section of the main coronaries but aberrant coronary arteries (often associated with dysrhythmias or sudden death) and saphenous vein bypass grafts (which are large and move little).

\section{Myocardial scar imaging}

Gadolinium is a paramagnetic MRI contrast agent that influences the magnetic relaxation times of local tissues, therefore altering their returning signal. Given intravenously, it redistributes into tissues and is safe to use in acute and chronic ischaemic heart disease. Areas of oedema after acute myocardial necrosis and scarring (chronic) have an excess of extracellular water producing a large volume of distribution

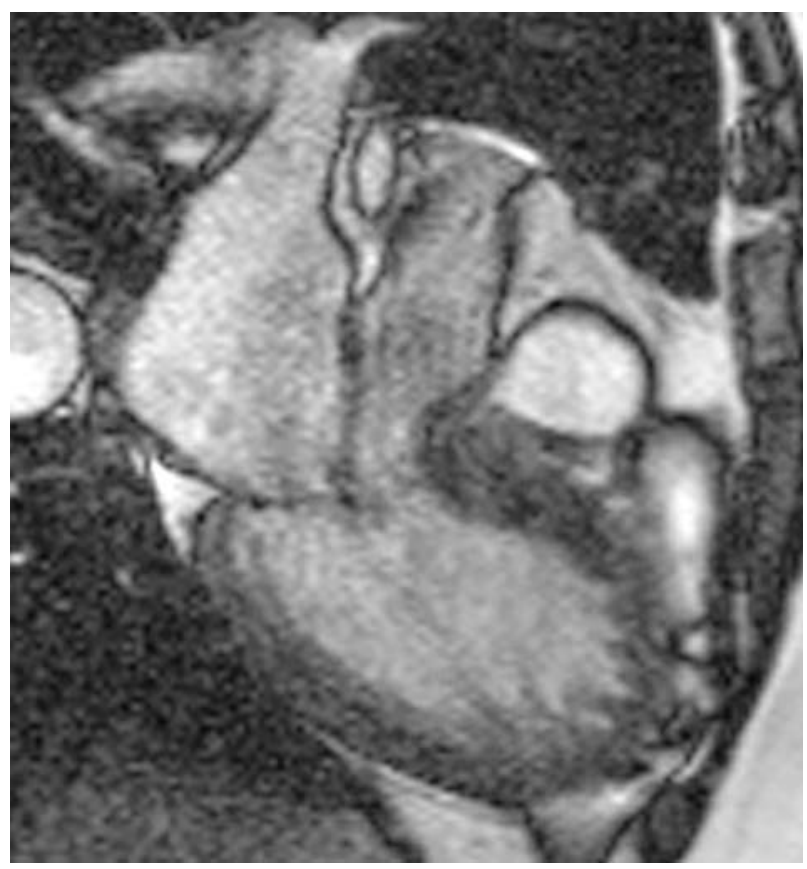

Figure 4 Hypertrophic obstructive cardiomyopathy. The asymmetrical septal thickening and obstruction to flow is clearly seen. into which the gadolinium accumulates. Early imaging after gadolinium injection shows enhancement of normal myocardium with darker areas of infarction due to poorer perfusion. Conversely, images taken 15 to 20 minutes later after gadolinium wash out from normal tissue show accumulation in the extracellular water of a myocardial scar, making old infarctions appear bright. A scar of $>50 \%$ of myocardial wall thickness has reduced chance of functional recovery after revascularisation. ${ }^{6}$

\section{Myocardial perfusion imaging}

Myocardial perfusion can be directly assessed by examining the rate of appearance of contrast agents in the myocardium. Reduced contrast enhancement of myocardium has been demonstrated in areas supplied with an artery of $>70 \%-80 \%$ stenosis, compared with areas with normal supply. ${ }^{7}$

\section{Stress imaging}

The principle employed by pharmacological stress echo imaging can be applied to cardiac MRI. The physiological effects of exercise are imitated by continuous infusion of a short acting sympathomimetic agent such as dobutamine. Myocardial wall motion is interrogated at rest and with different doses of dobutamine being infused. Worsening wall motion with increasing stress confirms the presence of functionally significant coronary artery disease. Sensitivity has been reported to be between $88 \%$ and $100 \%$. This technique can also be used to determine if hypokinetic myocardium is viable. ${ }^{8}$

\section{Aortography}

MRI is becoming the first line investigation in elective, noncoronary angiography of the aorta and central and peripheral vessels to assist in planning surgery. The aorta is easily imaged and, using multiplanar reformatting, the presence of aneurysms, dissections, and coarctation can be clearly delineated. MRI appears to be more sensitive and specific than transoesophageal echo or computed tomography for identifying acute dissection. ${ }^{9}$ Flow studies allow differentiation between fast flow in the true lumen, slow flow in the false lumen, intraluminal thrombus, and haematoma. MRI is also the investigation of choice in the long term follow up of coarctation and dissection repairs as reproducible, high quality anatomical views can be acquired without intraarterial access or radiation exposure.

\section{Tumours}

Three dimensional imaging by MRI allows precise definition of tumour size and site of attachment and identifies myocardial invasion. The intravascular space provides natural contrast to surrounding tissues. Tumour tissue can be characterised in terms of being solid or cystic, and its vascularity assessed. The larger field of view of MRI compared with echocardiography also allows assessment of the mediastinum, and pulmonary vessels, for more widespread disease. Blood clot within the cardiac chambers may also be identified because of a lowered signal due to the magnetic properties of haemosiderin.

\section{Congenital heart disease}

MRI is particularly helpful in patients who have undergone a corrective procedure. While it has been shown that transoesophageal echocardiography is better at fine detail, cardiac MRI excels in looking at conduits and flows. ${ }^{10}$ When used together, the need for invasive angiography is often removed. MRI is of particular value in the anatomical diagnosis and assessment of intracardiac shunting in septal defects, follow up of the right heart in repaired Fallot's tetralogy, transposition of the great vessels, and complex congenital heart 
disease. The multiplanar nature of MRI assists in clarifying abnormal anatomy and intracardiac shunts.

\section{Cardiomyopathy}

Echocardiography is the investigation of choice in the diagnosis of suspected hypertrophic cardiomyopathy but when image quality is poor or the diagnosis is in doubt, MRI can demonstrate the myocardium in three dimensions, outflow tract gradient and abnormal septal, longitudinal, and valvular motion.

Restrictive cardiomyopathy is characterised by impaired filling of the ventricles with raised filling pressures. Cardiac sarcoid, cardiac amyloid, iron overload, and endomyocardial fibrosis will produce an abnormal myocardial signal and help to exclude the main differential diagnosis of pericardial constriction.

MRI is more sensitive than echocardiography when imaging the pericardium for focal or generalised thickening.

One important cause of ventricular tachycardia (and sudden death) is arrythmogenic right ventricular cardiomyopathy. Localised fat infiltration may be an early feature with global thinning, ventricular dilatation, and dyskinesis and fatty infiltration occurring later. Endomyocardial biopsy can be hazardous and sampling error may be misleading giving a false negative result. Echocardiography may miss early changes that can be detected readily by MRI.

\section{CONCLUSIONS}

MRI is becoming more widely available and access to cardiac MRI will soon be offered in district general hospitals to compliment existing echocardiographic and other imaging modalities. It is well tolerated and safe, requiring nothing more invasive than a peripheral venous cannula. Absolute contraindications are few.

Ischaemic heart disease forms the bulk of the workload in most cardiology departments and although there are multiple imaging modalities already available, MRI often offers significant advantages. It could become a "one stop" cardiac imaging modality as a single examination (currently lasting around one hour) can assess structure, function, areas of infarction, and myocardial perfusion. It may soon be possible to provide anatomical information on coronary stenoses but also the physiological information regarding their functional importance. This has yet been clinically validated and is the subject of ongoing research.

\section{Authors' affiliations}

G J Heatlie, Queen's Medical Centre, Nottingham, UK

K Pointon, Department of Radiology, Nottingham City Hospital, Nottingham, UK

\section{REFERENCES}

1 Rogers WA, Pratt CM Weiner $\mathrm{DH}$, on behalf of the SOLVD investigators. Studies of left ventricular dysfunction. Am J Cardiol 1990;66:315-22.

2 Bellenger NG, Burgess M, Ray SG, et al on behalf of the CHRISTMAS Steering Committee and Investigators. Comparison of left ventricular ejection fraction and volumes in heart failure by two-dimensional echocardiography, radionuclide ventriculography and cardiovascular magnetic resonance: are they interchangeable? Eur Heart J 2000;21:1387-96.

3 Dulce MC, Mostbeck GH, O'Sullivan M, et al. Severity of aortic regurgitation: interstudy reproducibility of measurements with velocity encoded cine MR imaging. Radiology 1992;185:235-40.

4 Fayad ZA, Fuster V, Fallon JT, et al. Noninvasive in vivo human coronary artery lumen and wall imaging using black-blood magnetic resonance imaging. Circulation 2000;102:506-10.

5 Post JC, van Rossum AC, Bronzwaer JG, et al. Magnetic resonance angiography of anomalous coronary arteries. A new gold standard for delineating the proximal course? Circulation 1995;92:3163-71.

$6 \mathrm{Kim}$ RJ, Fieno DS, Parrish RB, et al. Relationship of MRI delayed contrast enhancement to irreversible injury, infarct age, and contractile function. Circulation 1999;100:185-92.

7 Panting JR, Gatehouse PD, Yang GZ, et al. Echo planar magnetic resonance myocardial perfusion imaging: parametric map analysis and comparison with thallium SPECT. J Magn Reson Imaging 2001;13:192-200.

8 Nagel E, Lehmkuhl HB, Bocksch W, et al. Noninvasive diagnosis of ischemia induced wall motion abnormalities with the use of high dose dobutamine stress MRI. Comparison with dobutamine stress echocardiography. Circulation 1999:99:763-70.

9 Nienaber CA, von Kodolitsch Y, Nicolas V, et al. The diagnosis of thoracic aortic dissection by non-invasive imaging procedures. $N$ Engl J Med 1993;328:1-9.

10 Hirsch R, Kilner PJ, Connelly M, et al. Diagnosis in adolescents and adults with congenital heart disease. Prospective assessment of the individual and combined roles of magnetic resonance imaging and transoesophageal echocardiography. Circulation 1994;90:2937-51. 